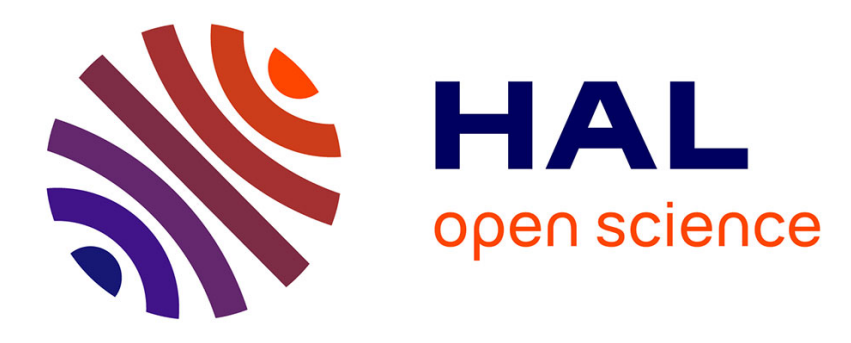

\title{
A Mathematical Analysis of the Cumulus Pricing Scheme
}

\author{
Yezekael Hayel, Bruno Tuffin
}

\section{To cite this version:}

Yezekael Hayel, Bruno Tuffin. A Mathematical Analysis of the Cumulus Pricing Scheme. [Research Report] RR-4500, INRIA. 2002. inria-00072088

\section{HAL Id: inria-00072088 https://hal.inria.fr/inria-00072088}

Submitted on 23 May 2006

HAL is a multi-disciplinary open access archive for the deposit and dissemination of scientific research documents, whether they are published or not. The documents may come from teaching and research institutions in France or abroad, or from public or private research centers.
L'archive ouverte pluridisciplinaire HAL, est destinée au dépôt et à la diffusion de documents scientifiques de niveau recherche, publiés ou non, émanant des établissements d'enseignement et de recherche français ou étrangers, des laboratoires publics ou privés. 
INSTITUT NATIONAL DE RECHERCHE EN INFORMATIQUE ET EN AUTOMATIQUE

\section{A Mathematical Analysis of the Cumulus Pricing Scheme}

Yezekael Hayel, Bruno Tuffin

\section{$\mathbf{N}^{\circ} 4500$}

Juillet 2002

THÈME 1 



\title{
A Mathematical Analysis of the Cumulus Pricing Scheme
}

\author{
Yezekael Hayel, ${ }^{*}$ Bruno Tuffin ${ }^{\dagger}$ \\ Thème 1 - Réseaux et systèmes \\ Projet Armor \\ Rapport de recherche $\mathrm{n}^{\circ} 4500$ - Juillet 2002 - 32 pages
}

\begin{abstract}
One important task in current and future communication networks is to define a suitable pricing scheme. It is then preferable to formulate a mathematical model, so that parameters will be optimized and importan$\mathrm{t}$ properties such as fairness or truthful anticipated load revelation will be verified. In this paper we study a simple and promising scheme called the Cumulus Pricing Scheme, which can address service differentiation and scalability among other issues. Our goal is to find out, from a mathematical model, parameter values optimizing the provider's revenue but under the constraint that each user has an incentive to reveal its anticipated load. This has led to a small variation of the initial model from the literature as, in our model, cumulus points are translated into financial terms, and measurements induce a cost as well.
\end{abstract}

Key-words: Cumulus Pricing Scheme, Network management, Quality-ofService.

(Résumé : tsvp)

\footnotetext{
* yhayel@irisa.fr
}

$\dagger$ btuffin@irisa.fr 


\section{Une analyse mathématique du modèle du Cumulus}

Résumé : Une des tâches parmi les plus importantes dans le domaine des futurs réseaux de communication est de définir un modèle de Tarification approprié. Il est alors préférable de formuler un modèle mathématique, afin d'optimiser les paramètres et de vérifier les propriétés importantes telles que l'équité ou l'incitation au respect de la déclaration anticipée de consommation. Dans ce rapport nous étudions un modèle simple et prometteur appelé le Cumulus, qui peut intégré de la différentiation de service et qui résiste au passage à l'échelle. Notre objectif est de d'obtenir, à partir d'un modèle mathématique, les paramètres du modèle qui optimisent le revenu du fournisseur sous la contrainte que chaque utilisateur indique sa futur consommation de façon objective. Ceci a conduit à une variation du modèle initial défini dans la littérature puisque, dans notre modèle les points du Cumulus sont traduits en terme financiers, ainsi que le coût lié aux mesures.

Mots-clé : Différenciation de services, modèle du Cumulus, gestion du réseau 


\section{Introduction}

The Internet is experiencing a tremendous growth of its traffic and a transformation of its architecture. The current flat-rate pricing scheme, adopted by most Internet Service Providers (ISPs), is an incentive to overuse the network which, in conjunction with the increasing number of subscribers, drives to congestion that reduces the Quality of Service (QoS). A usage-based charging scheme would overcome this drawback. Similarly, the future network architecture will have to respond to different QoS requirements of different types of applications. Architectures such as Diffserv proposal [8] deal with this problem, but an adapted pricing scheme has to be associated with, unless what a user would always choose the service class providing the best QoS.

Devising a new pricing scheme is the subject of an extensive research (see $[2,3,4,9,25,28,29]$ for introductory or overview papers). To our knowledge, the main streams about pricing schemes are the following:

- Charging models for guaranteed services such as voice or video should be related to connection acceptance control (CAC). This has been studied in $[6,21,31]$ for instance. In the Internet, the reservation of resources can be realized using RSVP [32]. Unfortunately, scalability issue is a major concern that has to be addressed when dealing with a Wide Area Network. That is why the next models do not include strict QoS guarantees but rather statistical QoS guarantees.

- Charging for elastic traffic based on transfer rates is one of the main schemes developed in the literature $[12,13,17,14]$ and can be adapted to the Internet. Either the user chooses the charge per unit time he will pay and his rate is determined by the network accordingly to a fairness criterion, or, other solution, he asks for a rate and a fair price is computed.

- Auctioning for bandwidth is also a possibility [15, 30]. Second price auction, where the "winner" does not pay its bid but the bid of the player he is excluding from the game, allow to obtain efficiency, convergence and incentive compatibility properties.

$\mathrm{RR} \mathrm{n}^{\circ} 4500$ 
- Priority pricing $[1,7,10,19,18]$ distinguishes different classes of traffic served with a given priority at each node of the network, a higher charge being associated to a greater priority. This model can be immediately related to Diffserv and can be decomposed into two subclasses: posted priority pricing where the price per class is fixed, and non-posted priority pricing where the prices depend on the level of congestion of the network.

- The proposal called Paris Metro Pricing (PMP) [20] is a simple alternative where the network is decomposed into several separate networks and each network, working like the current Internet, has a different connection fee so that we expect that the most expensive ones will not be less congested. Although very attractive, it is argued in [5] that PMP would not work under competition.

- The Cumulus Pricing Scheme (CPS) [22, 23] is also a simple possibility. A contract is negotiated between the ISP and the user. During periods of time, the utilization is measured and (positive or negative) cumulus points are awarded, depending on how far we are from the contract specifications. Threshold values of consumption fluctuations are used in order to award the points. After a while, the contract is renegotiated if the number of points exceeds a given value. In addition, at a given time, extra-fees can be charged.

The CPS is promising due to its simplicity and adaptability (to Diffserv architecture for instance), in part because it is applied at the edge of the network and because it is close to the flat-rate pricing. In this paper, we study the CPS from a mathematical point of view. Our contributions are the following:

- We have a deeper look at the economic model by studying, from the user and the network points of view, the benefits in terms of the threshold values. In our model, each cumulus point is translated into financial terms, as well as is the measurement procedure for the network.

- By a mathematical analysis, we then show that the initial CPS creates an incentive to "cheat", meaning to not reveal the anticipated resources consumption. 
- By a slight modification of the model, we give a sufficient condition on threshold values under which truthful revelation is the most profitable for the users. Remarkably, our sufficient condition is independent of the user's utility function, representing his valuation of the required service.

- Then, under this constraint, we give some elements helping the provider to set the threshold values in order to maximize its revenue. In a general setting, we claim that analytical results are intractable to obtain; we then use a simulated annealing algorithm. Nevertheless, in some particular cases, like with symmetric or linear thresholds, we have been able to obtain some analytical results.

The paper is organized as follows. In Section 2 we describe and analyze the CPS; Subsection 2.1 presents the model given in the literature; Subsection 2.2 gives some additional definitions of provider's revenue and user's overall level of satisfaction that will be helpful in our analysis; Subsection 2.3 shows that the CPS does not provide an incentive to reveal its real anticipated network consumption. Based on this result, we slightly modify the original CPS model in Section 3 by penalizing every overuse of the network. We then give some conditions over threshold values under which each user has no incentive to cheat, meaning an incentive to give the truthful estimate of its consumption. Under this condition, Section 4 works on setting threshold values maximizing the provider's revenue. Some numerical illustrations of our results are given in Section 5 and the conclusions and directions for future research are given Section 6.

\section{The Cumulus Pricing Scheme (CPS)}

In Subsection 2.1, we describe the Cumulus Pricing Scheme as devised in [22, 24, 23, 27]. Subsection 2.2 and 2.3 are new and study the truthful anticipated consumption revelation incentivity.

\subsection{Model presentation}

The CPS is characterized by its behavior at different time-scales: a contract is negotiated between the user and the provider, defining a flat-rate pricing;

$\mathrm{RR} \mathrm{n}^{\circ} 4500$ 
then the consumption is measured, verifying the contract's respect by assigning points. At the larger granularity, if the number of points exceeds a threshold over some periods, the contract is renegotiated.

Formally, define $V(t)$ as the actual resource consumption at time $t$. The time interval is decomposed in monitoring sub-intervals $\left[t_{i}, t_{i+1}\right](i \geq 1)$, so that, if the expected resource consumption (from the contract) is $x$, the overor underutilization over period $\left[t_{i}, t_{i+1}\right]$ is

$$
\Delta_{i}=\int_{t_{i}}^{t_{i+1}} V(t) d t-x\left(t_{i+1}-t_{i}\right) .
$$

At the end of each monitoring period, some cumulus points are assigned according to the following rule, where $N$ is the maximum number of points that can be assigned: define $\theta_{n}, n=-N, \cdots, N$ and $\theta_{ \pm(N+1)}= \pm \infty$ (with $\theta_{0}=0$ ) as thresholds such that $C_{i}:=C\left(\Delta_{i}\right)$ (positive or negative) points are assigned if

$$
0 \leq \theta_{C_{i}} \leq \Delta_{i} \leq \theta_{C_{i}+1} \text { or } \theta_{C_{i}-1} \leq \Delta_{i} \leq \theta_{C_{i}} \leq 0 .
$$

As a reaction rule, the provider decides to renegotiate the contract after the monitoring period $n$ as soon as

$$
\left|\sum_{i=1}^{n} C_{i}\right| \geq \Theta
$$

where $\Theta$ is another threshold.

The provider has to define a tariff function $p(x)$ per resource unit, when the user is requiring $x$ resource units. The total charge is then $c(x)=x p(x)$.

Assume that a user having required $x$ is actually consuming $\xi=x+\Delta$ (with $\Delta>0$ ). An extra fee is charged after accumulating a sufficient number of points, based on the estimated overuse $\delta$ for the period during which cumulus points have been accumulated (so $\delta=\bar{X}-x$ where $\bar{X}$ is the standard estimator of $\xi$ ). For convenience, function $c$ is used so that the additional charge is $c(\delta)$. Let

$$
\Psi(x, \xi)=c(\xi)-(c(x)+c(\xi-x))
$$

be the penalty function, being the over-charge for not telling the right requirement. The functions should verify the following properties : 
1. $\forall x>0, \quad p(x)>0$ is monotonically decreasing (economy of scale).

2. $\forall x>0, \quad c(x)=x p(x)$ is monotonically increasing (increasing cost).

3. if $x \neq \xi \quad \Psi(x, \xi)<0$ and $\Psi(x, \xi)=0$ if $x=\xi$.

4. $\Psi(x, x+\delta)$ is monotonically decreasing in $\delta$.

5. $|\Psi(x, \xi)|<|\Psi(\beta x, \beta \xi)| \leq \beta|\Psi(x, \xi)|$ for $\beta>1$.

For instance, $c(x)=\sqrt{x}$ fulfills these requirements [23].

With respect to the current Internet flat-rate pricing scheme, the CPS need$\mathrm{s}$ to measure the actual used resources. The precision of these measurements has to be good enough in order to limit the probability of wrongly assigning cumulus points. Assuming that $V(t)$ is a stochastic process in steady-state, from usual central limit theorem (with an unknown variance) using $n$ independent measurements, we can obtain a confidence interval with half-width $\varepsilon_{\alpha, n}=O(1 / \sqrt{n})$ with a given probability $\alpha$. In order to make the model more or less independent of the measurement method applied, two neighboring thresholds need to be at least at distance $2 \varepsilon_{\alpha, n}$.

\subsection{Mathematical definitions of revenues}

We are now going to define the network revenue and the user level of satisfaction. Our goal is get a deeper vision than in previous subsection, where we will be able to appreciate the economic impact of the threshold values. Before formally expressing the network revenue and user level of satisfaction, let us enumerate their components.

We assume that each cumulus point (positive or negative) has an economic effect for the user and the provider, directly or indirectly (for instance in the renegotiation procedure). We hope that this assumption (with respect to the general model defined in the previous sub-section) will provide some elements when defining threshold values. The economic impact of each point is expressed by a parameter $\gamma$. Thus, depending on the sign of $\delta$, the difference between the actual use and the contract, and whether you are a customer or the provider, the positive or negative cumulus points are translated into positive or negative financial impact.

$\mathrm{RR} \mathrm{n}^{\circ} 4500$ 
Another cost for the network comes from the measurement sample size $n$. Recall that in order to make the model more or less independent of the measurement method, $n$ must satisfy the following condition [23] :

$$
\forall i \in\{-N, \ldots N-1\}, \quad \theta_{i+1}-\theta_{i} \geq 2 \varepsilon_{\alpha, n}
$$

where $\varepsilon_{\alpha, n}=\frac{q_{\alpha} \hat{S}}{\sqrt{n}}$, with $\hat{S}$ the unbiased estimator of the variance of the stochastic process $V(t)$ and $q_{\alpha}$ the $\left(1-\frac{\alpha}{2}\right)$ quantile of the normal distribution. Condition (1) gives a minimum distance between two neighboring thresholds which leads to the following condition over $n$,

$$
n \geq \frac{4 \hat{S}^{2} q_{\alpha}^{2}}{\min \left(\theta_{i+1}-\theta_{i}\right)^{2}} .
$$

Introducing the parameter $\beta$ as the economic impact of one measurement and assuming that the sampling cost is linear with respect to $n$ gives a measurement cost $\beta n$.

We introduce a last cost for the provider which depends on the measurement error estimation. It seems relevant to penalize the provider with this kind of "ethical" cost from the precision of the model. We suppose that this cost is linear with the length of the confidence interval which is $2 \varepsilon_{\alpha, n}=2 \frac{\hat{S} q_{\alpha}}{\sqrt{n}}$.

So we use the function $C_{n e t}$ to express the network costs depending on the sample size $n$,

$$
C_{n e t}(n)=\beta n+2 \mu \frac{\hat{S} q_{\alpha}}{\sqrt{n}} .
$$

The network's profits come from the total charge $c(x)$ for the demand in the SLA and the extra fee $c(\delta)$ from the overconsumption if the specific renegotiation threshold $\Theta$ is reached and, of course, the cumulus points if the user under-estimates his consumption. So, for the $k^{\text {th }}$ monitoring period (which is a month for example) between two renegociations, the network revenue for one user is expressed by the following definition:

Definition 1 The expression of the network revenue for one user who requires $x$ but consumes $x+\delta_{k}$ is

$$
G_{n e t}\left(x, \delta_{k}, \theta, k, n\right)=c(x)+c\left(\sum_{i=1}^{k} \delta_{i}\right) \mathbb{1}_{\left\{\sum_{i=1}^{k} C_{i} \geq \Theta\right\}}+\gamma C\left(\delta_{k}\right)-C_{n e t}(n) .
$$


In Definition 1 , we can remark that $\theta$ is in $\mathbb{R}^{2 N}, \delta_{k}$ is a real representing the difference between actual and from contract consumption, $\Theta$ is the number of cumulative points for giving the extra fee and the renegociation, and $x$ is the expected resource consumption as specified in the contract.

Like in usual economic modeling theory, each user is characterized by an increasing utility function $U(x)$ (expressed in financial terms) of getting $x$ as the expected resource consumption. His costs depend on the expected resource requirement, the extra consumption and the positive cumulus points.

Definition 2 The level of satisfaction of a user, or his benefit, of requiring $x$ but consuming $x+d$ for the $k^{\text {th }}$ period is

$$
G_{u}\left(x, \delta_{k}, \theta, k\right)=U\left(x+\delta_{k}\right)-c(x)-\gamma C\left(\delta_{k}\right)-c\left(\sum_{i=1}^{k} \delta_{i}\right) \mathbb{1}_{\left\{\sum_{i=1}^{k} C_{i} \geq \Theta\right\}}
$$

Note that the discrete random variable $C_{i}$ depends on the thresholds $\theta$ and on $\delta_{i}$.

\subsection{About cheating incentivity}

The assumed role of the penalty function $\Psi$ is to prevent the user from an a priori over- or under-estimation of his use by the point 3. of its properties, because the model must restrain the user to respect his contract, as this kind of attitude penalize the ISP for capacity planning. But the translation into financial terms of measurements and of cumulus points require further analysis. The following definition describes how a user has an incentive to truthfully reveal his anticipated consumption.

Definition 3 The property of truthful anticipated consumption revelation of the model is expressed by the following condition:

$\forall \theta, k \quad \exists x^{*}>0$ such that

$$
\arg \max _{x, \delta} G_{u}(x, \delta, \theta, k)=\left(x^{*}, 0, \theta, k\right)
$$

$\mathrm{RR} \mathrm{n}^{\circ} 4500$ 
This means that a customer chooses his consumption in order to maximize his level of satisfaction and, if the model behaves correctly, the expected consumption is the one which maxims his level of satisfaction. Now, we present an important result on the CSP model about this condition of truthful revelation.

Theorem 1 The model of the Cumulus Pricing Scheme defined in this section doesn't verify the propriety of truthful revelation of anticipated consumption expressed in Definition 3.

We prove this theorem in the appendix, part A, by showing that in a particular user behaviour, Condition (3) is not satisfied. Specifically, it's not verified since the customer can cheat by a small variation $\delta \in\left[0, \theta_{1}[\right.$ so that he will never be penalized by a cumulus point.

Remark 1 First, note that, interestingly, the result from this theorem is independent of the utility function. Second, note that this behaviour is restraining in the case of a wide network with a big number of consumers. In the following proposition, we present a sufficient condition that constraints users to not cheat over the first threshold $\theta_{1}$.

Proposition 1 If the positive thresholds verify the following equations:

- $\theta_{1}(x)<c^{-1}(\gamma+c(x))-x$,

- for $k \geq 2, \quad \theta_{k}(x)<c^{-1}((k-1) \gamma+c(x))-x$,

then:

$\forall \theta, k, x>0, \delta_{k} \in\left[\theta_{1},+\infty\left[\right.\right.$, we have $G_{u}\left(x, \delta_{k}, \theta, k\right)<G_{u}\left(x+\delta_{k}, 0, \theta, k\right)$.

This proposition implies that a big overuse is avoided. It is proved in part B of the appendix by observing the evolution of the difference between the two behaviors.

In the next part, we modify the model in order to generate incentives for users to reveal exactly their anticipated consumption. Particularly, they will not even have an interest to cheat under the first threshold $\theta_{1}$. 


\section{The total penalty CPS}

Thus, in order to prevent the cheating situation, we introduce a small variation of the model described above. It consists in charging the penalty $c(\delta)$ at the end of each period instead of just when a number $\Theta$ of points have been assigned. This is a particular case of the precedent model where the variable $\Theta$ is fixed to 0 but where, differently, we charge as soon as there is an overconsumption, i.e $\delta>0$. Then, the definitions of the network revenue and the level of satisfaction of the user need to be modified. We present them in the next sub-section.

\subsection{Definitions}

Let us define, in this new context, the network revenue and user level of satisfaction for any periods of measurements. They are here easier to understand as the penalty is applied at each of these periods.

Definition 4 For any period of measurements, the network revenue for a user who requires $x$ and consumes $x+\delta$ is:

$$
G_{n e t}(x, \delta, \theta, n)=c(x)+c(\delta) \mathbb{1}_{\{\delta>0\}}+\gamma C(\delta)-C_{n e t}(n),
$$

and the level of satisfaction of one user is:

$$
G_{u}(x, \delta, \theta)=U(x+\delta)-c(x)-c(\delta) \mathbb{1}_{\{\delta>0\}}-\gamma C(\delta) .
$$

The (simple) difference in the model, with respect to the one of Section 2 allows to avoid cheating incentivity because the penalty is charged at the end of each period of measurements. We prove this result in the next section.

\subsection{About cheating incentivity for the new model}

In this part, we analyze the property of truthful revelation of anticipated consumption of Definition 3 for this second model. This model based on a penalty applied at the end of each period can constrain the users to respect their contract. We formulate a sufficient condition to prove this result in the next theorem.

$\mathrm{RR} \mathrm{n}^{\circ} 4500$ 
Theorem 2 The total penalty Cumulus Pricing Scheme satisfies the truthful revelation of anticipated consumption property if the negative thresholds verify

$$
\forall i \geq 1 \quad \text { and } \quad x>0, \quad c\left(\theta_{-i}+x\right)<c(x)-i \gamma .
$$

This theorem is shown in the part $\mathrm{C}$ of the Appendix. Note that this sufficient condition deals with the negative thresholds only. It is very interesting because it is independent of the utility function. Nevertheless, it is not a unique sufficient condition. For instance, another condition could be, for threshold $\theta_{-i}: \forall i>0, \forall x>0, \forall \delta \in[-x,+\infty[\backslash\{0\}$,

$$
U\left(\theta_{-i}+x\right)-U(x)-c(\delta) \mathbb{1}_{\{\delta>0\}}-\gamma C(\delta)<0 .
$$

This result comes from the important fact that the level of satisfaction for a user who respects its contract must be greater than the one of someone who does not do it for the same anticipated consumption, i.e.,

$$
G_{u}(x, \delta, \theta)-G_{u}(x, 0, \theta)<0, \quad \forall \theta, \delta, x .
$$

Another important remark about our result on thresholds is the dependence between the thresholds and the contract specification $x$. This fact has an effect on the optimization of the network revenue.

\section{Optimizing the provider's revenue}

\subsection{Optimization problem and minimization of the cost function $C_{n e t}$}

We have seen what condition on the negative thresholds the model can satisfy to verify the property of truthful anticipated consumption revelation. Our goal now is to find out the configuration of thresholds optimizing the total revenue of the network, under the constraint of Theorem 2.

In the previous subsection, we have defined the network revenue and the user level of satisfaction. We consider that users come in the network with a 
mean rate $\lambda$ which is a function of the average level of satisfaction introduced in Definition 2. So, we define the total network revenue by:

$$
\int_{0}^{+\infty} \mathbb{E}\left(G_{n e t}(x, \delta, \theta, n)\right) d \lambda\left(\mathbb{E}\left(G_{u}(x, \delta, \theta)\right)\right) .
$$

We also have seen in the previous sub-section a sufficient condition for the property of truthful revelation of anticipated consumption (where the thresholds depend on the resource required $x$ ) which means that either a customer enters the network at its anticipated consumption $x$, or let him out if it is too expensive for him. Anyway, he has no interest of entering at another declared level $x^{\prime}$. Thus, the maximization of the expression above is equivalent to maximize the product

$$
\lambda\left(\mathbb{E}\left(G_{u}(x, \delta, \theta)\right)\right) \mathbb{E}\left(G_{n e t}(x, \delta, \theta, n)\right)
$$

for all $x$, subject to the constraint

$$
\forall i \geq 1 \quad \text { and } \quad x>0, \quad c\left(\theta_{-i}(x)+x\right)<c(x)-i \gamma
$$

This is a problem under an inequality constraint.

Assumption 1 We will assume from now and throughout the paper that the difference $\delta$ between the actual and the anticipated consumption is a random variable. Following the truthful revelation of anticipated consumption property, it is reasonable to assume that the density of $\delta$ is symmetric, so that its mean is zero.

To maximize the network revenue given by Equation (4), the provider has first to minimize the cost function $C_{n e t}$ under the constraint (2). This function has a minimum in

$$
n^{*}=\left(\frac{\hat{S} q_{\alpha} \mu}{\beta}\right)^{2 / 3}
$$

so we can define the relation between $n$ and the thresholds. If $n^{*}$ is smaller than $\frac{4 \hat{S}^{2} q_{\alpha}^{2}}{\min \left(\theta_{i+1}-\theta_{i}\right)^{2}}$ (i.e., constraint (2)), then the minimum is obtained for $n=n^{*}$, otherwise for $n=\frac{4 \hat{S}^{2} q_{\alpha}^{2}}{\min \left(\theta_{i+1}-\theta_{i}\right)^{2}}$. So we can actually express the cost function in

$\mathrm{RR} \mathrm{n}^{\circ} 4500$ 
terms of the thresholds by:

$$
\begin{aligned}
C_{n e t}\left(\min \left(\theta_{i+1}-\theta_{i}\right)\right)= & K \mathbb{1}_{\left\{\min \left(\theta_{i+1}-\theta_{i}\right) \geq \kappa\right\}}+ \\
& \left(4 \beta \frac{\hat{S}^{2} q_{\alpha}^{2}}{\min \left(\theta_{i+1}-\theta_{i}\right)^{2}}+\mu \min \left(\theta_{i+1}-\theta_{i}\right)\right) \mathbb{1}_{\left\{\min \left(\theta_{i+1}-\theta_{i}\right)<\kappa\right\}},
\end{aligned}
$$

with

$$
\kappa=2\left(\frac{\beta}{\mu}\right)^{1 / 3}\left(\hat{S} q_{\alpha}\right)^{2 / 3}
$$

and

$$
K=3 \beta^{1 / 3}\left(\mu \hat{S} q_{\alpha}\right)^{2 / 3} .
$$

As $n$ is actually a function of $\theta$, we will write, in all following equations, $G_{n e t}(x, \delta, \theta)$ instead of $G_{n e t}(x, \delta, \theta, n)$.

\subsection{Optimization in a general setting}

The difficulty with the optimization stems from the non-derivability of function $C_{n e t}(\theta)$. Thus, we need to resort to numerical approaches. In this paper, we use a simulated annealing algorithm. This class of continuous global optimization algorithms seems the most adapted for our objective function because, first, this method requires no specifications of derivability and, second, it is specifically adapted to functions with multiple local optima. The algorithm we use is called ASA for Adaptive Simulated Annealing [16]. It is probably the most widely tested SA algorithm in the literature, and its code is publicly available at the web site http://www.ingber.com. We use more specifically ASAMIN which is a gateway function to ASA developed by S. Sakata [26]. One particularity of this algorithm is the use of the temperature to define the densities of the next candidate point [11].

In Section 5, we illustrate numerically this method on some examples. But, under some restrictions, we are able to derive $C_{n e t}(\theta)$; we present now some results in these particular positions of thresholds. 


\subsection{Analytic results in particular cases}

\subsubsection{Symmetric thresholds}

We consider the configuration proposed by Reichl et al. in [23, 27] where the thresholds verify for all $k, \theta_{k}=-\theta_{-k}$. Hence the mean number of assigned cumulus points $\in\{0, \pm 1, \ldots, \pm N\}$ is zero, and the thresholds intervene only in the cost function of the network's revenue $C_{n e t}$ for our optimization. Maximizing the total network revenue is equivalent to maximizing the network revenue for one user, which is then itself equivalent to minimizing the cost function $C_{n e t}(\theta)$ in terms of the minimum of difference between two neighboring thresholds.

Thus, to optimize the total network revenue, we have to set the thresholds so that the minimum difference is more than $\kappa$ (given by Equation (6)) and also so that the negative thresholds satisfy the sufficient condition defined in Theorem 2. Note that in this case, the total network revenue is independent of the number of thresholds (as it actually depends only on the minimum difference between successive thresholds), so we can take like Reichl et al. in [23], 3 to max 5 on each direction for simplicity. We illustrate this numerically in Section 5.

\subsubsection{Linear thresholds}

Let us now consider another case where thresholds are linear, i.e.,

$$
\forall i>0, \quad \theta_{i+1}-\theta_{i}=\theta_{+} \quad \text { and } \quad \theta_{-i}-\theta_{-i-1}=\theta_{-} .
$$

Our optimization is then restricted to the variables : $\theta_{+}$and $\theta_{-}$which are respectively the distance between positive thresholds and between negative ones. Those variables are positive real numbers. The mean number of cumulative points obtained by a user expressed in terms of those variables is:

$$
\begin{aligned}
\mathbb{E}(C(\delta)) & =\sum_{k=1}^{N} k\left(F_{\delta}\left(\theta_{k+1}\right)-F_{\delta}\left(\theta_{k}\right)\right)+\sum_{k=1}^{N}(-k)\left(F_{\delta}\left(\theta_{-k}\right)-F_{\delta}\left(\theta_{-k-1}\right)\right), \\
& =N-\sum_{k=1}^{N} F_{\delta}\left(k \theta_{+}\right)-\sum_{k=1}^{N} F_{\delta}\left(-k \theta_{-}\right),
\end{aligned}
$$

$\mathrm{RR} \mathrm{n}^{\circ} 4500$ 
where $F_{\delta}$ is the cumulative distribution function of $\delta$ (its density is denoted by $\left.f_{\delta}\right)$ and where $\theta_{k}=k \theta_{+}$if $k>0, k \theta_{-}$otherwise.

1) $\theta_{+}<\theta_{-}$On one hand, suppose that $\theta_{+}<\theta_{-}$. So the cost of measurements depends on $\theta_{+}$(see Section 4.1). Note that, to simplify the expression, we will write $\lambda$ and $\mathbb{E}\left(G_{n e t}\right)$ without their arguments in all following equations. The first-order conditions of optimization problem are then:

$$
\begin{aligned}
\frac{\partial}{\partial \theta_{-}}\left(\lambda \mathbb{E}\left(G_{n e t}\right)\right) & =\mathbb{E}\left(G_{n e t}\right) \lambda^{\prime} \gamma \sum_{k=1}^{N}(-k) f_{\delta}\left(-k \theta_{-}\right)-\lambda \gamma \sum_{k=1}^{N}(-k) f_{\delta}\left(-k \theta_{-}\right), \\
& =0
\end{aligned}
$$

and

$$
\begin{aligned}
\frac{\partial}{\partial \theta_{+}}\left(\lambda \mathbb{E}\left(G_{n e t}\right)\right) & =\mathbb{E}\left(G_{n e t}\right) \lambda^{\prime} \gamma \sum_{k=1}^{N} k f_{\delta}\left(k \theta_{+}\right)-\lambda \gamma \sum_{k=1}^{N} k f_{\delta}\left(k \theta_{+}\right)-\lambda \frac{\partial}{\partial \theta_{+}} C_{n e t}\left(\theta_{+}\right), \\
& =0 .
\end{aligned}
$$

Subtracting both equations, we get:

$$
\frac{\partial}{\partial \theta_{+}} C_{n e t}\left(\theta_{+}\right)=\left(-8 \beta \frac{\hat{S}^{2} q_{\alpha}^{2}}{\theta_{+}^{3}}+\mu\right) \mathbb{1}_{\left\{\theta_{+}<\kappa\right\}}=0 .
$$

So we find the solution $\theta_{+}=\kappa$ like in Section 4.1. Then, inserting $\theta_{+}^{*}=\kappa$ in the other order condition, we can find $\theta_{-}^{*}$, after factorization Equation (8), as the result of equation:

$$
\begin{array}{r}
\left(D-\sum_{k=1}^{N} F_{\delta}\left(-k \theta_{-}\right)\right) \cdot \lambda^{\prime}\left(\mathbb{E}(U(x+\delta))-D-K+\sum_{k=1}^{N} F_{\delta}\left(-k \theta_{-}\right)\right) \\
=\lambda\left(\mathbb{E}(U(x+\delta))-D-K+\sum_{k=1}^{N} F_{\delta}\left(-k \theta_{-}\right)\right) .
\end{array}
$$

with $D=c(x)+\mathbb{E}\left(c(\delta) \mathbb{1}_{\{\delta>0\}}\right)+\gamma N-\sum_{k=1}^{N} F_{\delta}(k \kappa)-K$.

2) $\theta_{-}<\theta_{+}$Suppose now that $\theta_{-}<\theta_{+}$. We use the same idea than in the previous case to derive a solution. The minimum between consecutive thresholds is $\theta_{-}$and, inverting the roles of $\theta_{+}$and $\theta_{-}$, we get:

$$
\frac{\partial}{\partial \theta_{-}} C_{n e t}\left(\theta_{-}\right)=0,
$$


and thus $\theta_{-}=\kappa$. To find now $\theta_{+}$, we have just to switch $\theta_{+}$and $\theta_{-}$in Equation (9).

3) $\theta_{-}=\theta_{+}$This is the sub-space where the function $C_{n e t}$ is not derivable because of the minimum function. But, in this case, thresholds are symmetric; we are then in the previous particular case and the solution gives $\theta_{+}$or $\theta$ equal to $\kappa$. Numerically, we compare the solution in this case with those obtained on $\Omega_{1}$ and $\Omega_{2}$.

Example with $N=1$ and $\lambda(x)=\sqrt{x}$ : Consider the simple case where $N=1$ and the rate of user's arriving is a square root function. The expression of the mean number of obtained points is:

$$
\mathbb{E}(C(\delta))=1-F_{\delta}\left(\theta_{+}\right)-F_{\delta}\left(-\theta_{-}\right)
$$

Denote by $\Omega_{1}$ the space where $\theta_{+}<\theta_{-}$and by $\Omega_{2}$ the other one. On $\Omega_{1}$, we have a solution $\left(\theta_{+}^{*}, \theta_{-}^{*}\right)$ like described above with $\theta_{+}^{*}=\kappa$ and $\theta_{-}^{*}$ solution of Equation (9):

$$
\theta_{-}^{*}=-F_{\delta}^{-1}\left(c(x)+\mathbb{E}\left(c(\delta) \mathbb{1}_{\{\delta>0\}}\right)+1-F\left(\theta_{+}^{*}\right)-\frac{2}{3} U(x+\delta)\right) .
$$

On $\Omega_{2}$, we have a solution $\left(\theta_{+}^{*}, \theta_{-}^{*}\right)$ with $\theta_{-}^{*}=\kappa$ and

$$
\begin{aligned}
\theta_{+}^{*} & =F_{\delta}^{-1}\left(c(x)+\mathbb{E}\left(c(\delta) \mathbb{1}_{\{\delta>0\}}\right)+1-F_{\delta}\left(\theta_{+}^{*}\right)-\frac{2}{3} U(x+\delta)\right) \\
& =-\theta_{-}^{*} .
\end{aligned}
$$

As $\theta_{-}^{*}$ and $\theta_{+}^{*}$ are positive reals, only one of those solutions is possible.

\subsubsection{Uniform thresholds}

We consider here that we have $N_{+}$positive thresholds and $N_{-}$negative and that the thresholds are distributed according to the quantiles of the random variable $\delta$, so that the probability to obtain one or more positive points is equal, as well as for the negative case. We can then express the thresholds by:

$$
\theta_{k}=\left\{\begin{array}{lll}
F_{\delta}^{-1}\left(\frac{1}{2}+\frac{k}{2\left(N_{+}+1\right)}\right) & \text { if } & k>0, \\
F_{\delta}^{-1}\left(\frac{1}{2}+\frac{k}{2\left(N_{-}+1\right)}\right) & \text { if } & k<0 .
\end{array}\right.
$$

$\mathrm{RR} \mathrm{n}^{\circ} 4500$ 
As we assume that the distribution of $\delta$ is symmetric, the expression of the mean number of assigned points is, from (7),

$$
\mathbb{E}(C(\delta))=\frac{\gamma}{4} N_{+}-\frac{\gamma}{4} N_{-} .
$$

The optimization problem is then defined in terms of the two parameters $N_{+}$and $N_{-}$. Consider the specific uniform distribution on the interval $[-Z, Z]$ as an example. In this case, the expression of the thresholds is:

$$
\theta_{k}=\left\{\begin{array}{l}
\frac{Z}{N_{+}+1} \quad \text { if } \quad k>0, \\
-\frac{Z}{N_{-}+1} \quad \text { if } \quad k<0 .
\end{array}\right.
$$

To get the maximum, we look at the first order conditions like in the previous example. Also, we consider that $N_{+}$and $N_{-}$are real in order to get the derivatives.

1) $N_{+}>N_{-}$On one hand, we assume that $N_{+}>N_{-}$and then the minimum of the difference between consecutive thresholds is $\theta_{1}$. Like in the previous example, we can get:

$$
\frac{\partial}{\partial N_{+}} C_{n e t}\left(\theta_{1}\left(N_{+}\right)\right)=0
$$

with the expression of $\theta_{1}$ described above. With this equation we found that:

$$
\theta_{1}=\kappa, \text { so that } N_{+}=\frac{Z}{\kappa}-1=N_{+}^{*}
$$

To obtain the second variable, we use the other first-order condition. We have the expression of the mean network revenue:

$$
\begin{aligned}
\mathbb{E}\left(G_{n e t}(x, \delta, \theta)\right)= & c(x)+\mathbb{E}\left(c(\delta) \mathbb{1}_{\{\delta>0\}}\right)+\frac{\gamma}{4} N_{+}^{*} \\
& -\frac{\gamma}{4} N_{-}-K, \\
= & A-\frac{\gamma}{4} N_{-},
\end{aligned}
$$

and the mean arrival rate for a user: $\lambda\left(\mathbb{E}\left(G_{\text {user }}(x, \delta, \theta)\right)\right)=$ $\lambda\left(\mathbb{E}(U(x+\delta))-c(x)-\mathbb{E}\left(c(\delta) \mathbb{1}_{\{\delta>0\}}\right)-\frac{\gamma}{4} N_{+}^{*}+\frac{\gamma}{4} N_{-}\right)$, 
$=\lambda\left(\mathbb{E}(U(x+\delta))-A-K+\frac{\gamma}{4} N_{-}\right)$.

The first order condition with $N_{-}$is:

$$
\frac{\partial}{\partial N_{-}} \lambda \mathbb{E}\left(G_{n e t}\right)=\mathbb{E}\left(G_{n e t}\right) \lambda^{\prime}(.)-\lambda(.)=0 .
$$

If we suppose that the arrival rate is expressed by the function $\lambda(x)=\sqrt{x}$, we have:

$$
\begin{array}{r}
\frac{\gamma}{4}\left(A-\frac{\gamma}{4} N_{-}\right) \frac{1}{2 \sqrt{\mathbb{E}(U(x+\delta))-A-K+\frac{\gamma}{4} N_{-}}} \\
-\sqrt{\mathbb{E}(U(x+\delta))-A-K+\frac{\gamma}{4} N_{-}}=0
\end{array}
$$

and then

$$
N_{-}=\frac{16}{8 \gamma+\gamma^{2}}\left(\frac{9 \gamma}{4} A-2 \mathbb{E}(U(x+\delta))+2 K\right)
$$

2) $N_{-}>N_{+}$In this case, we use exactly the same procedure. The difference is that the minimum difference between consecutive thresholds is $\theta_{-1}$. So the result we obtain for the optimization is:

$$
\theta_{-1}=\kappa,
$$

coming from the equation:

$$
\frac{\partial}{\partial N_{-}} C_{n e t}\left(\theta_{-1}\left(N_{-}\right)\right)=0
$$

We obtain $N_{-}^{*}=\frac{Z}{\kappa}-1$, like the solution for the number of positive thresholds in the previous case. Using the other first-order condition, we have:

$$
N_{+}=\frac{4}{3 \gamma}\left(2 \mathbb{E}(U(x+\delta))+3 K-3 A^{\prime}\right)
$$

with $A^{\prime}=c(x)+\mathbb{E}\left(c(\delta) \mathbb{1}_{\{\delta>0\}}\right)-\frac{\gamma}{4} N_{-}^{*}-3 \beta^{1 / 3}\left(\hat{S} q_{\alpha} \mu\right)^{2 / 3}$.

$\mathrm{RR} \mathrm{n}^{\circ} 4500$ 
3) $N_{+}=N_{-}$As in the previous example, this case implies that the thresholds are symmetric. The solution occurs in this case when the minimum of difference between consecutive thresholds is equal or superior to $\kappa$. For our example here, in terms of the number of positive and negative thresholds, the maximum is obtained when $N_{+}=N_{-}=\frac{Z}{\kappa}-1$.

Remark 2 To find the maximum between each above possible solution, we compare them numerically.

\section{$5 \quad$ Numerical illustrations}

In this section, we present some illustrations of the results that can be obtained. First, we are interested in the user's level of satisfaction and the incentive to cheat; we also compare the user's level of satisfaction for the two CPS models. Second, we observe the results obtained with the simulated annealing algorithm and we discuss the evolution of the total network revenue with the number of thresholds. Finally, we look at the results obtained in the particular cases defined in Section 4.3.

In all the following examples, we use the following functions:

- the demand function $\lambda(y)=\sqrt{y}$,

- the utility function $U(x)=10 \sqrt{x} e^{-\frac{x}{2 X}}+\sqrt{x}$ where $X$ is a given parameter.

\subsection{About cheating incentivity}

We present here an example in order to study the property of truthful anticipated consumption revelation. We propose to illustrate the difference of level of satisfaction between a user who requires $x$ and uses $x+\delta$ and another who wants $x+\delta$ and just uses it with these two models. It is important to note that we present results with the first model where $\Theta$ is very large, to have the worth case for the network revenue and to use sufficient condition of Proposition 1.

The results are displayed in Figure 1. We consider the thresholds $\theta=$ $[-40,-20,0,10,18,40,60,90]$ which verify the sufficient condition on the positive thresholds for the initial model (Proposition 1) and the other one on the 


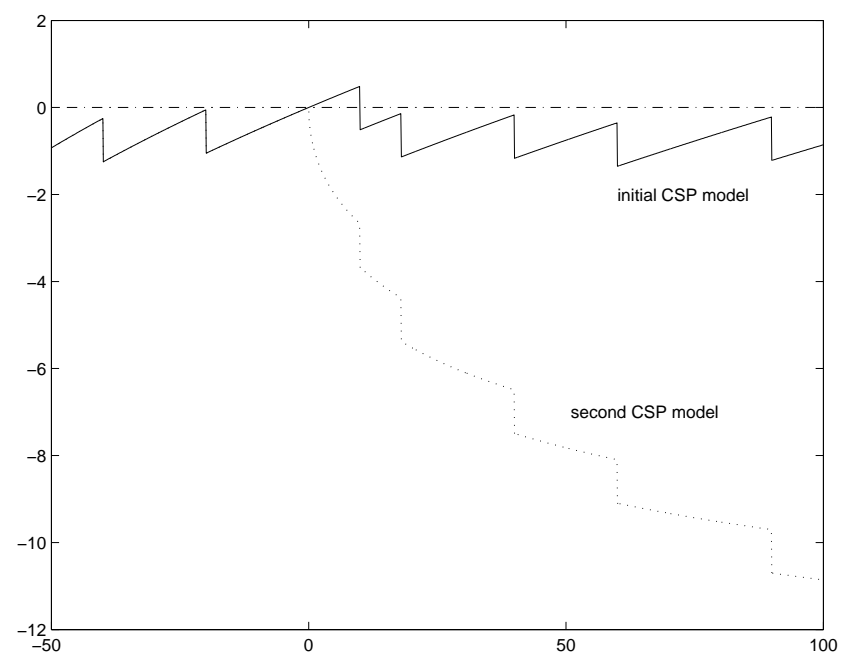

Figure 1: Evolution of $G_{u}(x, \delta, \theta)-G_{u}(x+\delta, 0, \theta)$ in terms of $\delta$.

negative thresholds for the second model (Theorem 2). The first sufficient condition gives:

$$
\theta_{1}<21, \theta_{2}<21, \theta_{3}<44, \theta_{4}<69 \text { and } \theta_{5}<96 \text {. }
$$

The second one gives:

$$
\theta_{-1}<-19 \text { and } \theta_{-2}<-36
$$

The user specified demand is $x=100$ and the cost by point is $\gamma=1$. We remark that, for the initial CPS model, the difference of level of satisfaction cannot be negative when the overuse $\delta$ is under the first threshold $\theta_{1}$. This illustrates the fact that this model doesn't have the property of truthful anticipated consumption revelation. But we see that for the second one, this difference is always negative. Furthermore, the difference is much larger in the case of overuse for the total penalty CPS, which is a desirable situation preventing more from cheating.

$\mathrm{RR} \mathrm{n}^{\circ} 4500$ 


\subsection{Simulated Annealing for general case optimization}

One important assumption is about the distribution of $\delta$. Because of the property of truthful revelation of anticipated consumption, we assume that the mean difference is zero. In addition, we suppose that the distribution is symmetric. We consider a Gaussian distribution with mean 0 and variance 40. In Table 1, we give the results obtained in this case.

\begin{tabular}{|c|c|c|}
\hline $\begin{array}{c}\text { Number } \\
\text { of } \\
\text { thresholds }\end{array}$ & Thresholds Positions & $\begin{array}{c}\text { Total } \\
\text { Network } \\
\text { Revenue }\end{array}$ \\
\hline 1 & -16.25 & 69.11 \\
\hline 2 & $-16.25,3.61$ & 190.47 \\
\hline 3 & $-16.25,4.15,8.31$ & 208.01 \\
\hline 4 & $-16.25,4.24,8.49,12.74$ & 209.55 \\
\hline 5 & $-13.3,4.06,8.12,12.19,16.25$ & 208.53 \\
\hline 6 & $-16.25,-12.70,-9.16,3.54,7.09,10.63,14.18$ & 203.33 \\
\hline 7 & $-16.23,-10.98,-7.58,3.25,6.50,9.75,13,16.25$ & 199.13 \\
\hline 8 & $-16.25,-13.20,-10.15,-7.11,3.04,6.10,9.14$ & 195.52 \\
\hline 9 & $12.20,15.25$ & \\
\hline 10 & $-13.75,-5.17,-1.96,1.17,3.53,4.68,8.85,11.05$ & 42.29 \\
& $13.35,14.67$ & \\
\hline
\end{tabular}

Table 1: Thresholds maximizing the total network revenue as obtained with the simulated annealing algorithm

In our algorithm, we have fixed the number of thresholds from 1 to 10 and the best threshold values have been determined each time (but note that the number of positive or negative thresholds is not fixed). If we compare each case, we can observe that the maximum revenue is obtained when there is four thresholds, one being negative and three being positive.

In order to verify the relevance of our results, we have also plotted the revenue in the case with only two thresholds, one positive and one negative, 
in Figure 2. We can verify that the value obtained in Table 1 (second line) is the actual maximum.

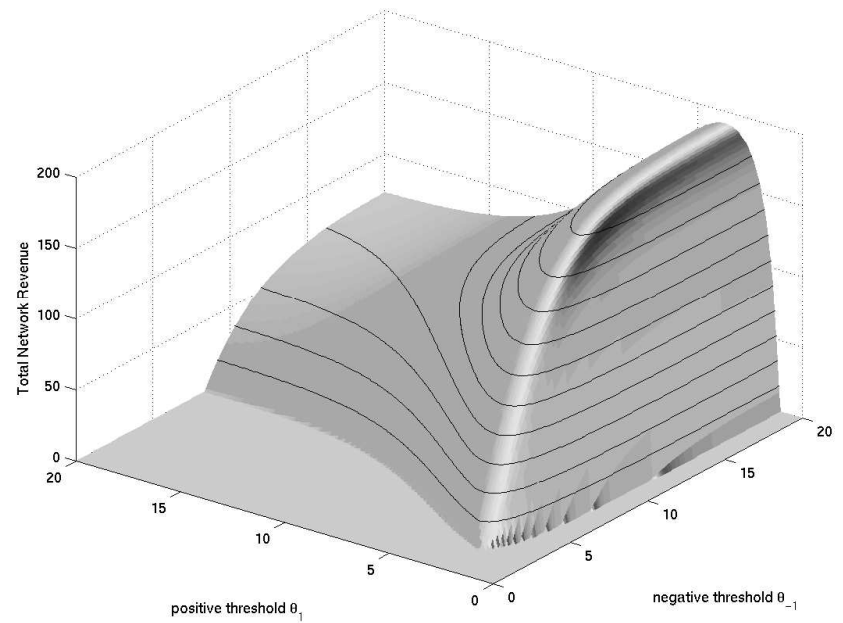

Figure 2: Network total revenue when there are two varying thresholds: one positive and one negative.

\subsection{Particular cases}

In this subsection, we illustrate the results obtained when the thresholds are placed in a special way. We choose the user's required consumption $x=100$, economic cost by cumulus point $\gamma=100$, cost for measurement $\beta=0.1$ and error cost $\mu=0.1$.

\subsubsection{Symmetric Thresholds}

In this special case, the total network revenue is expressed in terms of the minimum distance between consecutive thresholds. In Figure 3, we plot the total network revenue in terms of the required quantity of resources $x$. The vertical line on the right represents the value $\kappa$ (from Equation (6)) and we see that behind this frontier, the total network revenue is constant. We remark as well that, as it could be expected, the revenue is increasing with $x$.

$\mathrm{RR} \mathrm{n}^{\circ} 4500$ 


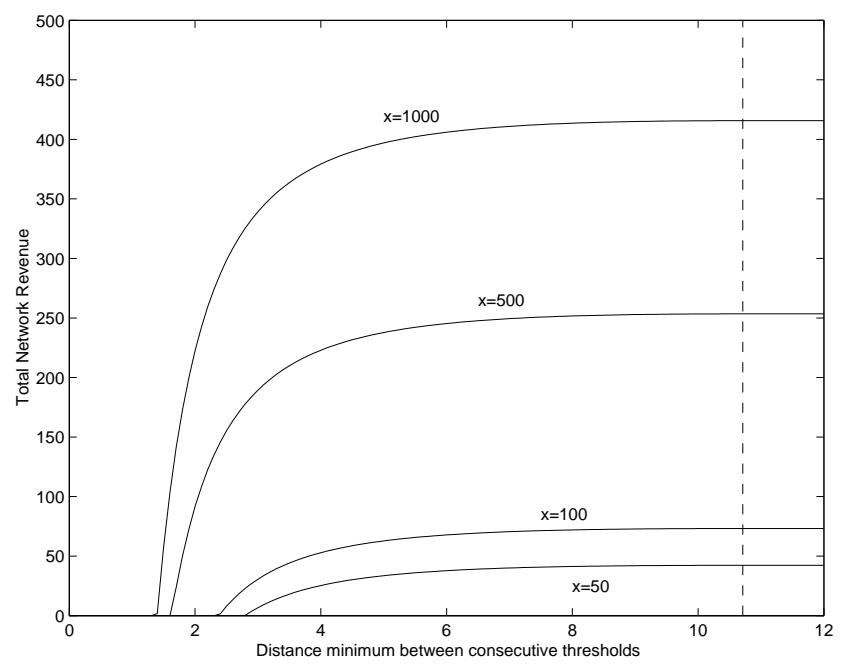

Figure 3: Network total revenue with symmetric thresholds in terms of the minimum distance between consecutive thresholds.

\subsubsection{Linear Thresholds}

We verify now our theoretical results from Section 4.3.2 with some particular values of parameters. We choose five positive thresholds and five negative.

With this configuration, we obtain that $\kappa=10.71$. We observe in Figure 4 , that the total network revenue is constant when $\theta_{+}$and $\theta_{-}$are above $\kappa$, because when the minimum is larger than $\kappa$, the cost of the network $C_{n e t}$ is independent of the thresholds. The maximum is reached when the distance between two successive negative thresholds is equal to $\kappa$ in sub-space $\Omega_{2}$. We remark also that the solution in the other derivability space $\Omega_{1}$ described in Section 4.3.2 is negative. This result verifies what we have shown in Section 4.3.2.

\subsubsection{Uniform threshold}

For this numerical illustration, we use the same variables as defined above except that $\beta=0.05$ and $\mu=5$. We display in Figure 5 the total network revenue with a varying number of positive and negative thresholds. The distribution of $\delta$ is uniform over the interval $[-50,50]$. 


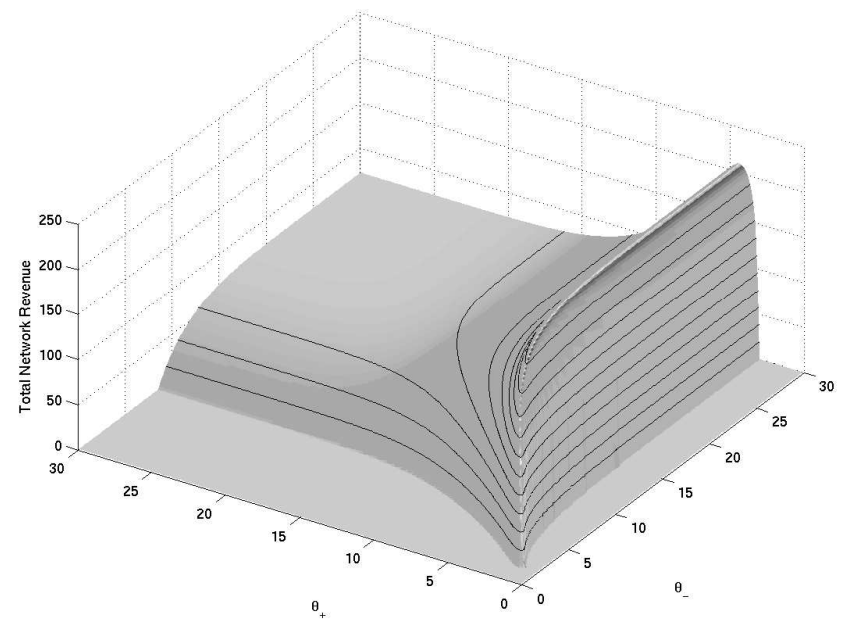

Figure 4: Network total revenue with linear thresholds in terms of the distance between neighboring positive thresholds $\theta_{+}$and negative $\theta_{-}$.

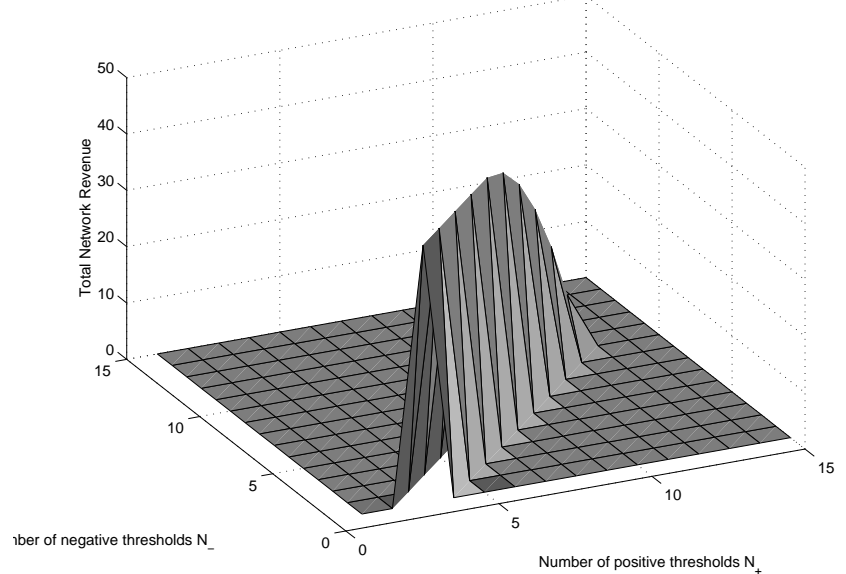

Figure 5: Network total revenue with uniform thresholds in terms of the number of positive threshold $N_{+}$and negative ones $N_{-}$.

As we can remark, we have only one optimum solution which is the same than given by Equations $(10,11)$ :

$$
N_{+}^{*}=6.82 \quad \text { and } \quad N_{-}^{*}=2.92 .
$$

$\mathrm{RR} \mathrm{n}^{\circ} 4500$ 


\section{Conclusions}

This paper was motivated by the parameters optimization problem for the Cumulus Pricing Scheme. First, after proving that, when points and measurements are financially translated, that the initial CPS provides an incentive to cheat, we have slightly modified the model to circumvent this problem. Second, we have obtained some results for optimizing the provider's revenue for this modified model in particular cases of thresholds which are:

- symmetric: thresholds verify for all $k>0, \theta_{k}=-\theta_{-k}$,

- linear: distances between the neighboring thresholds is equal,

- uniform: the probability to obtain one or more points is the same and identically for the negative points.

In a general case, we have used a simulated annealing algorithm to optimize the total network revenue in terms of position of the thresholds.

As future directions of research, one can be interested in one particularity of measurement: the granularity. How behaves the model if the cumulus points are assigned every second or every hour or every month? We could also extend the model by including service differentiation, or parameters of traffic like delay, jitter, etc. Finally, we could analyze the CPS under competition between ISPs.

\section{References}

[1] R. Cocchi, D. Estrin, S. Shenker, and L. Zhang. Pricing in Computer Networks: Motivation, Formulation and Example. IEEE/ACM Transactions on Networking, 1(6):614-627, 1993.

[2] L.A. DaSilva. Pricing of QoS-Enabled Networks: A Survey. IEEE Communications Surveys \& Tutorials, 3(2), 2000.

[3] P. Dolan. Internet Pricing. is the end of the World Wide Wait in view? Communications \& Strategies, 37:15-46, 2000. 
[4] M. Falkner, M. Devetsikiotis, and I. Lambadaris. An Overview of Pricing Concepts for Broadband IP Networks. IEEE Communications Surveys \& Tutorials, 3(2), 2000.

[5] R. Gibbens, R. Mason, and R. Steinberg. Internet service classes under competition. IEEE Journal on Selected Areas in Communications, 18(12):2490-2498, 2000.

[6] R.J. Gibbens, S.K. Sargood, F.P. Kelly, H. Azmoodeh, R. Macfadyen, and N. Macfadyen. An Approach to Service Level Agreements for IP networks with Differential Services.

[7] A. Gupta, D.O. Stahl, and A.B. Whinston. Priority Pricing of Integrated Services Networks. In Lee W. McKnight and Joseph P. Bailey, editors, Internet Economics, pages 323-352. MIT Press, 1997.

[8] J. Heinanen, F. Baker, W. Weiss, and J. Wroclawski. Assured forwarding phb group. IETF: RFC 2597, 1999.

[9] T. Henderson, J. Crowcroft, and S. Bhatti. Congestion Pricing. Paying Your Way in Communication Networks. IEEE Internet Computing, September/October:85-89, 2001.

[10] M.L. Honig and K. Steiglitz. Usage-based pricing of packet data generated by a heterogeneous user population. In Proceedings of IEEE INFOCOM 95, pages 867-874, 1995 .

[11] L. Ingber. Simulated Annealing: Practice versus Theory. Mathematical Computing Modelling, 18(11):29-57, 1993.

[12] F.P. Kelly. Mathematical modelling of the Internet. In Proceedings of the Fourth International Congress on Industrial and Applied Mathematics, 2000 .

[13] F.P. Kelly, A.K. Mauloo, and D.K.H. Tan. Rate control in communication networks: shadow prices, proportional fairness and stability. Journal of the Operational Research Society, 49:237-252, 1998.

$\mathrm{RR} \mathrm{n}^{\circ} 4500$ 
[14] R.J. La and V. Anantharam. Window-Based Control with Heterogeneous Users. In Proceedings of IEEE INFOCOM 2001, 2001.

[15] A.A. Lazar and N. Semret. Design and Analysis of the Progressive Second Price Auction for Network Bandwidth Sharing. To appear in Telecommunication Systems, 13, 2001. http://comet.columbia.edu/〜nemo/ telecomsys.pdf.

[16] M. Locatelli. Simulated Annealing Algorithms for Continuous Global Optimization. Journal of Optimization Theory and Applications, 104:121$133,2000$.

[17] S.H. Low and D.E. Lapsley. Optimization Flow Control, I: Basic Algorithm and Convergence. IEEE/ACM Transactions on Networking, 7(6), 1999.

[18] P. Marbach. Pricing Differentiated Services Networks: Bursty Traffic. In Proceedings of IEEE INFOCOM 2001, 2001.

[19] H. Mendelson and S. Whang. Optimal incentive-compatible priority pricing for the M/M/1 queue. Operations Research, 38(5):870-883, 1990.

[20] A. Odlyzko. Paris Metro Pricing for the Internet. In ACM Conference on Electronic Commerce (EC'99), pages 140-147, 1999.

[21] I.Ch. Paschalidis and J.N. Tsitsiklis. Congestion-Dependent Pricing of Network Services. IEEE/ACM Transactions on Networking, 8(2):171184,2000 .

[22] P. Reichl, P. Flury, J. Gerke, and B. Stiller. How to overcome the feasibility problem for tariffing internet services: the cumulus pricing scheme. In Proceedings of IEEE ICC 2001, vol. 7, pages 2079-2083, 2001.

[23] P. Reichl and B. Stiller. Edge pricing in space and time: Theoretical and practical aspects of the cumulus pricing scheme. In Proceedings of the 17th International Teletraffic Congress, 2001. 
[24] P. Reichl and B. Stiller. Nil nove sub sole: Why internet charging schemes look like as they do. In Proceedings of the 4 th Berlin Internet Economic Workshop, 2001.

[25] J.W. Roberts. Quality of Service Guarantees and Charging in Multiservice Networks. IEICE Trans. Commun., E81(5):824-831, 1998.

[26] S. Sakata. Asamin, 2002. http://www.econ.1sa.umich.edu/ ${ }^{\text {ssakata/ }}$ software.

[27] B. Stiller, J. Gerke, P. Reichl, and P. Flury. Management of Differentiated Services Usage by the Cumulus Pricing Scheme and a Generic Internet Charging System. In Proceedings of the Symposium on Integrated Network Management, 2001.

[28] B. Stiller, P. Reichl, and S. Leinen. Pricing and Cost Recovery for Internet Services: Practical Review, Classification, and Application of Relevant Models. Netnomics, 2(1), 2000.

[29] B. Tuffin. Charging the Internet without bandwidth reservation: an overview and bibliography of mathematical approaches. Technical Report 1434, IRISA, January 2002.

[30] B. Tuffin. Revisited Progressive Second Price Auctions for Charging Telecomunication Networks. Telecommunication Systems, page To appear, 2002 .

[31] Q. Wang, J.M. Peha, and M.A. Sirbu. Optimal Pricing for Integrated Services Networks. In Lee W. McKnight and Joseph P. Bailey, editors, Internet Economics, pages 353-376. MIT Press, 1997.

[32] L.S. Zhang, D. Deering, S. Shenker, and D. Zappala. RSVP: A resource ReSerVation Protocol. IEEE Network Magazine, 1993.

\section{A Proof of Theorem 1}

The customer's expected resource consumption expressed in the contract is $x$. Suppose that a user consumes exactly the same quantity of resource $x+\delta_{k}$

$\mathrm{RR} \mathrm{n}^{\circ} 4500$ 
during each period. Then

$$
\begin{aligned}
G_{u}\left(x, \delta_{k}, \theta_{k}, k\right)= & U\left(x+\delta_{k}\right)-c(x)-\gamma C\left(\delta_{k}\right) \\
& -c\left(\sum_{i=1}^{k} \delta_{i}\right) \mathbb{1}_{\left\{\sum_{i=1}^{k} C_{i} \geq \Theta\right\}} .
\end{aligned}
$$

To prevent the hysteresis effect of the measure, the first threshold $\theta_{1}$ is different from zero. Suppose that $\delta_{k}$ is between 0 and $\theta_{1}$. A necessary condition to obtain Theorem 2 is that $\forall k, \theta, \exists x>0, \forall \delta_{k} \in[-x,+\infty[\backslash\{0\}$,

$$
G_{u}\left(x-\delta_{k}, \delta_{k}, \theta, k\right)<G_{u}(x, 0, \theta, k),
$$

meaning that the user benefits from respecting his contract for the same actual consumption. This condition is easily verified from the definition of truthful anticipated consumption revelation of Equation (3). As $\delta_{k}<\theta_{1}, C\left(\delta_{k}\right)=0$, thus $\sum_{i=1}^{k} C_{i}=0$ and

$$
\begin{aligned}
G_{u}\left(x-\delta_{k}, \delta_{k}, \theta, k\right)-G_{u}(x, 0, \theta, k) & =c(x)-c\left(x-\delta_{k}\right) \\
& >0,
\end{aligned}
$$

which shows that the necessary condition (12) is not verified.

Thus, in this particularly situation, the user has an incentive to cheat, so that the property of truthful anticipated consumption revelation is not verified by the model.

\section{B Proof of Proposition 1}

Consider $x>0$ and $\delta_{k}>\theta_{1}$. The expression of the difference of benefits, for an anticipated belief of consumption $x+\delta_{k}$, between a cheating declaration $x$ and a truthful revelation $x+\delta_{k}$ is:

$G_{u}\left(x, \delta_{k}, \theta, k\right)-G_{u}\left(x+\delta_{k}, 0, \theta, k\right)$

$\left.=c\left(x+\delta_{k}\right)-c(x)-\gamma C\left(\delta_{k}\right)-c \sum_{i=1}^{k} \delta_{i}\right) \mathbb{1}_{\left\{\sum_{i=1}^{k} C_{i} \geq K\right\}}$. We want to prove that this difference is negative so a sufficient condition is that the function

$$
D\left(\delta_{k}\right)=c\left(x+\delta_{k}\right)-c(x)-\gamma C\left(\delta_{k}\right)
$$


is negative (this expression is when the penalization is not applied, this is the worst case for network revenue).

First we have a sufficient condition for the first threshold $\theta_{1}$ given by the following in-equation:

$$
D\left(\theta_{1}\right)=c\left(x+\theta_{1}\right)-c(x)-\gamma<0,
$$

from the assumptions. Second, we look at the other thresholds. The function $D$ is strictly increasing with $\delta_{k}$ when $\delta_{k} \in\left[\theta_{k-1}, \theta_{k}[\right.$. It is then sufficient to look if the condition is verified when $\delta_{k}$ tends to $\theta_{k}$. Formally:

$$
\begin{aligned}
\lim _{\varepsilon \rightarrow 0} D\left(\theta_{k}-\varepsilon\right) & =D\left(\theta_{k}\right)+\gamma \\
& =c\left(x+\theta_{k}\right)-c(x)-\gamma(k-1) .
\end{aligned}
$$

Thus, we have a sufficient condition on positive thresholds to ensure that a user should not cheat by more than $\theta_{1}$.

\section{Proof of Theorem 2}

Lemma 1 A sufficient condition for truthful anticipated consumption revelation for the total penalty $C P S$ is $\forall x>0, \forall \delta \in[-x,+\infty[\backslash\{0\}$,

$$
c(x+\delta)-c(x)-c(\delta) \mathbb{1}_{\{\delta \geq 0\}}-\gamma C(\delta)<0 .
$$

This lemma comes from the fact that for all $x$ and $\delta$, the difference between the level of satisfaction when a user requires $x$ and consumes $x+\delta$, and a user who requires $x+\delta$ and respects its contract, is negative, i.e.,

$$
\forall \delta, x, \theta \quad G_{u}(x, \delta, \theta)-G_{u}(x+\delta, 0, \theta)<0 .
$$

Then if we replace the expression of the level of satisfaction from Definition 4, we immediately get Lemma 1.

Proof of Theorem 2: We separate the case of overuse and underuse, i.e., $\delta$ positive or negative. For all $k$ and $\theta$, in the case where $\delta$ is positive we have

$\mathrm{RR} \mathrm{n}^{\circ} 4500$ 


$$
\begin{aligned}
G_{u}(x, \delta, \theta, k)-G_{u} & (x+\delta, 0, \theta, k) \\
& =c(x+\delta)-c(x)-c(\delta)-\gamma C(\delta), \\
& \leq \Psi(x, x+\delta)<0 .
\end{aligned}
$$

Thus, from the properties of function $\Psi$, this difference is strictly negative when we have an overuse.

Next, for $x>0$ and $\delta \in[-x, 0$ [ (i.e., negative), if the thresholds verify

$$
\forall i \geq 1, \quad c\left(x+\theta_{-i}\right)<c(x)-i \gamma
$$

we have $G_{u}(x, \delta, \theta, k)<G_{u}(x+\delta, 0, \theta, k)$. So, if this condition is verified, we have: $\forall x>0, \forall \delta \in[-x,+\infty[\backslash\{0\}$,

$$
c(x+\delta)-c(x)-c(\delta) \mathbb{1}_{\{\delta \geq 0\}}-\gamma C(\delta)<0,
$$

which gives the result from Lemma 1. 


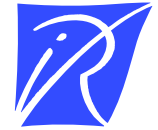

Unité de recherche INRIA Lorraine, Technopôle de Nancy-Brabois, Campus scientifique, 615 rue du Jardin Botanique, BP 101, 54600 VILLERS LÈS NANCY

Unité de recherche INRIA Rennes, Irisa, Campus universitaire de Beaulieu, 35042 RENNES Cedex

Unité de recherche INRIA Rhône-Alpes, 655, avenue de l'Europe, 38330 MONTBONNOT ST MARTIN

Unité de recherche INRIA Rocquencourt, Domaine de Voluceau, Rocquencourt, BP 105, 78153 LE CHESNAY Cedex

Unité de recherche INRIA Sophia-Antipolis, 2004 route des Lucioles, BP 93, 06902 SOPHIA-ANTIPOLIS Cedex

Éditeur

INRIA, Domaine de Voluceau, Rocquencourt, BP 105, 78153 LE CHESNAY Cedex (France)

http://www.inria.fr

ISSN 0249-6399 